Article

\title{
Remote Phonon Scattering in Two-Dimensional InSe FETs with High- $\kappa$ Gate Stack
}

\author{
Pengying Chang * $\mathbb{0}$, Xiaoyan Liu *, Fei Liu and Gang Du \\ Institute of Microelectronics, Peking University, Beijing 100871, China; fliu003@gmail.com (F.L.); \\ gangdu@pku.edu.cn (G.D.) \\ * Correspondence: pychang@pku.edu.cn (P.C.); xyliu@ime.pku.edu.cn (X.L.); Tel.: +86-152-1038-8557 (P.C.)
}

Received: 15 November 2018; Accepted: 17 December 2018; Published: 19 December 2018

check for updates

\begin{abstract}
This work focuses on the effect of remote phonon arising from the substrate and high- $\kappa$ gate dielectric on electron mobility in two-dimensional (2D) InSe field-effect transistors (FETs). The electrostatic characteristic under quantum confinement is derived by self-consistently solving the Poisson and Schrödinger equations using the effective mass approximation. Then mobility is calculated by the Kubo-Greenwood formula accounting for the remote phonon scattering (RPS) as well as the intrinsic phonon scatterings, including the acoustic phonon, homopolar phonon, optical phonon scatterings, and Fröhlich interaction. Using the above method, the mobility degradation due to remote phonon is comprehensively explored in single- and dual-gate InSe FETs utilizing $\mathrm{SiO}_{2}$, $\mathrm{Al}_{2} \mathrm{O}_{3}$, and $\mathrm{HfO}_{2}$ as gate dielectric respectively. We unveil the origin of temperature, inversion density, and thickness dependence of carrier mobility. Simulations indicate that remote phonon and Fröhlich interaction plays a comparatively major role in determining the electron transport in InSe. Mobility is more severely degraded by remote phonon of $\mathrm{HfO}_{2}$ dielectric than $\mathrm{Al}_{2} \mathrm{O}_{3}$ and $\mathrm{SiO}_{2}$ dielectric, which can be effectively insulated by introducing a $\mathrm{SiO}_{2}$ interfacial layer between the high- $\kappa$ dielectric and InSe. Due to its smaller in-plane and quantization effective masses, mobility begins to increase at higher density as carriers become degenerate, and mobility degradation with a reduced layer number is much stronger in InSe compared with $\mathrm{MoS}_{2}$.
\end{abstract}

Keywords: two-dimensional material; field effect transistor; indium selenide; phonon scattering; mobility; high- $\kappa$ dielectric

\section{Introduction}

The compelling demand for higher performance and lower power consumption in complementary metal-oxide-semiconductor (CMOS) field-effect transistors (FETs) has highlighted the quest for devices and architectures based on new materials [1]. Performance boosters such as strain, high- $\kappa$ dielectric, metal gate, and three-dimensional (3D) devices have enabled extraordinary improvement of performance in the past 60 years [2,3]. Recently, two-dimensional (2D) van der Waals semiconductors hold great potential for optics and electronics application due to their unique properties, including the atomic thickness, tunable bandgap, and dangling-bond-free surface, which achieves improved gate control over the channel and reduced short channel effects $[4,5]$. So far, many classes of 2D material-based devices have been extensively studied, such as graphene, transition metal dichalcogenides (TMDs), and black phosphorus [6-8]. Very recently, few-layer InSe has attracted much attention due to its highly promising prospect as channel material for FETs, offering small effective mass of electron $\sim 0.14 m_{0}$ and high electron mobility up to $\sim 10^{3} \mathrm{~cm}^{2} / \mathrm{Vs}$ at room temperature obtained by experimental measurements [9-11]. Therefore, InSe has advantages of a similar gap as silicon, 2D nature as graphene, higher mobility than TMDs, and higher environmental stability than black 
phosphorus. In addition, electrostatic tunability of spin-orbit coupling in InSe has been identified, showing potential in devising III-VI based spintronic devices [12,13].

However, the charge transport properties in InSe FET have not been well understood and starve for comprehensive investigation. More recently, the ballistic performance of mono- and multi-layer InSe FET is studied by the first-principles calculation and the top of the barrier model [14], and temperature-dependent phonon-limited mobility is estimated by the physical modeling of intrinsic scattering mechanisms [15]. On the other hand, charge transport behavior is very sensitive to external surroundings, such as gaseous adsorbates from air and trapped charges in substrates [16], and their electronic performance is generally lower than their intrinsic values. Previous studies of back-gated multilayer InSe FET on various substrates (bare $\mathrm{SiO}_{2}$, bare $\mathrm{Al}_{2} \mathrm{O}_{3}$, poly(methyl methacrylate) (PMMA) $/ \mathrm{SiO}_{2}$, and PMMA $/ \mathrm{Al}_{2} \mathrm{O}_{3}$ ) have reported the carrier mobility ranging from $2.2 \mathrm{~cm}^{2} / \mathrm{Vs}$ to $1055 \mathrm{~cm}^{2} /$ Vs at low operating voltage [10], while dual-gated InSe FET on hexagonal boron nitride (hBN) $/ \mathrm{SiO}_{2}$ show an excellent mobility approaching $10^{3} \mathrm{~cm}^{2} / \mathrm{Vs}$ and $10^{4} \mathrm{~cm}^{2} / \mathrm{Vs}$ at room and liquid-helium temperatures respectively [9]. It is apparently suggested that the introduction of substrate and gate dielectric has a strong effect on the electron mobility, which can be generally contributed to the extrinsic scatterings from surface roughness (SRS), interfacial Coulomb impurities (CIS), and remote phonon scatterings (RPS) $[17,18]$. Atomic flatness of 2D materials makes them immune to SR scattering, while CIS can be lowered or eliminated as possible by improving the fabrication process. Therefore, only remote phonon can be regarded as an intrinsic factor arising from the dielectric environment, and open questions remain as to its role in determining the electron transport in atomically-thin InSe FETs.

In this paper, the effect of remote phonons arising from the substrate and high- $\kappa$ dielectric together with the intrinsic phonons of the InSe channel on electron transport is studied based on the physical modeling by self-consistently solving the Poisson and Schrödinger equations and employing the Kubo-Greenwood formula. Mobility behaviors in single-gate and dual-gate InSe FET with various gate dielectric are theoretically explored and analyzed as a function of temperature, inversion density, InSe layer number, and $\mathrm{SiO}_{2}$ interfacial layer thickness. Acoustic phonons and optical phonons-as well as homopolar phonons-have a minor effect on electron mobility, while remote phonons and Fröhlich interaction play a comparatively major role in determining the electron transport in InSe. Compared with $\mathrm{MoS}_{2}$, much smaller effective masses of electron in InSe give rise to a great enhancement of mobility at high density as carriers become degenerate. Simulation results in this work provide physical insight into the mobility behavior of InSe FET for carrier mobility optimization from the theoretical viewpoint.

This paper is organized as follows. Section 2 describes the device structures and simulation methods, especially the physical models of remote phonon depending on the gate stack. In Section 3, we present simulation results of mobility and corresponding explanations. Finally, the conclusion is drawn in Section 4.

\section{Device Structures and Simulation Methods}

Simulated device structures with 2D-layered InSe channel are shown in Figure 1, where the intrinsic channel without doping is assumed. Figure 1a shows the back-gate (single-gate) InSe FET with $\mathrm{SiO}_{2}$ substrate as gate dielectric. Figure $1 \mathrm{~b}$ shows the top-gate (dual-gate) InSe FETs with high- $\mathcal{K}$ dielectric as top dielectric and $\mathrm{SiO}_{2}$ substrate as back dielectric. Figure 1c shows the structure with additional $\mathrm{SiO}_{2}$ interfacial layer (ITL) embedded between the InSe channel and high- $\kappa$ dielectric compared with Figure $1 \mathrm{~b}$. In the case of single-gate devices, only the back gate is biased with $\mathrm{V}_{\mathrm{bg}}$, while the back gate is grounded and the top gate is biased with $\mathrm{V}_{\mathrm{tg}}$ for the dual-gated devices. In this work, traditionally used high- $\kappa$ dielectrics of $\mathrm{HfO}_{2}$ and $\mathrm{Al}_{2} \mathrm{O}_{3}$ are comprehensively studied, with corresponding parameters listed in Table 1. Except for Figure 2, all the simulation results are calculated at room temperature $(300 \mathrm{~K})$. 
We start the calculation by obtaining the electrostatic characteristic of the two-dimensional electron gas (2DEG) in InSe layer by self-consistently solving the Poisson and Schrödinger equations using the effective mass approximation with nonparabolicity correction, inherently accounting for the quantum confinement effects [19]. Particularly the energy dispersion of 2D-layered InSe is described by the thickness-dependent effective masses obtained from first-principles calculation, as shown in our previous work [14]. Next, the matrix elements and the scattering rates are calculated through the Fermi golden rule [19]. Physical models for electron mobility include the remote phonon scattering (RPS) arising from the high- $\kappa$ dielectric as well as the intrinsic phonon scatterings of channel material, including the acoustic (AC) phonon-, homopolar (HO) phonon-, optical (OP) phonon- scatterings, and Fröhlich interaction (POP) [20-23]. For AC phonons, elastic and isotropic approximations are adopted. The HO and OP scatterings are treated as inelastic and isotropic process. For POP and RP scatterings, inelastic and anisotropic characteristic are considered. Once the scattering rates are obtained, the mobility is calculated by the Kubo-Greenwood formula employing the momentum relaxation time approximation. The parameters for mobility calculation in few-layer InSe are taken from our previous work [15].

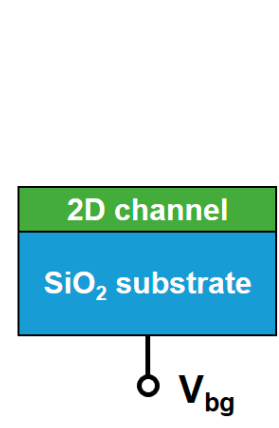

(a) Device A

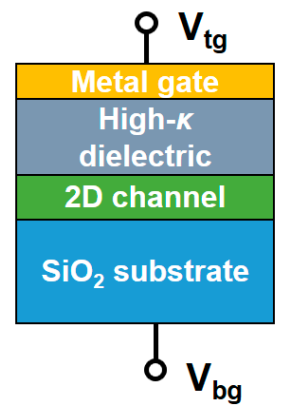

(b) Device B

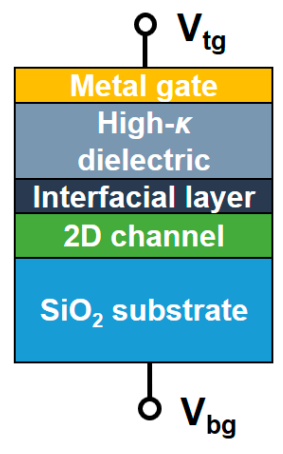

(c) Device C

Figure 1. Simulated device structure with atomically thin InSe channel in this work. (a) Back-gate InSe field-effect transistors (FET) with $\mathrm{SiO}_{2}$ substrate as gate dielectric. (b) Dual-gate InSe FET with high- $\kappa$ dielectric as top-gate dielectric and $\mathrm{SiO}_{2}$ substrate as back-gate dielectric. (c) The same structure as (b) with additional $\mathrm{SiO}_{2}$ interfacial layer embedded between the InSe channel and high- $\kappa$ dielectric. For the dual-gate structure, high- $\kappa$ dielectric is covered with a metal gate.

Table 1. Parameters for the polar phonons in some high- $\kappa$ materials [20]

\begin{tabular}{cccc}
\hline Quantity & $\mathbf{S i O}_{\mathbf{2}}$ & $\mathbf{A l}_{\mathbf{2}} \mathbf{O}_{\mathbf{3}}$ & $\mathbf{H f O}_{\mathbf{2}}$ \\
\hline$\varepsilon_{0}$ & 3.90 & 12.53 & 22.00 \\
$\varepsilon_{\text {int }}$ & 3.05 & 7.27 & 6.58 \\
$\varepsilon_{\infty}$ & 2.50 & 3.20 & 5.03 \\
$\hbar \omega_{T O 1}$ & 55.60 & 48.18 & 12.40 \\
$\hbar \omega_{T O 2}$ & 138.10 & 71.41 & 48.35 \\
\hline
\end{tabular}

$\varepsilon_{0}$ : Static (low-frequency) dielectric constant; $\varepsilon_{\text {int }}$ : dielectric constant at an intermediate frequency; $\varepsilon_{\infty}$ : high-frequency dielectric constant; $\hbar_{\omega_{T O}}, \hbar_{\omega_{T O} 2}$ : frequencies of the two polar phonons.

For remote phonon induced by $\mathrm{SiO}_{2}$ substrate, the semi-infinite substrate is assumed, and the dispersion relationship for the remote phonon model can be written as [19]

$$
\omega_{R P}=\omega_{\mathrm{TO}_{1, \mathrm{SiO}_{2}}} \sqrt{\frac{\varepsilon_{\mathrm{InSe}}+\varepsilon_{\mathrm{SiO}_{2}, 0}}{\varepsilon_{\mathrm{InSe}}+\varepsilon_{\mathrm{SiO}_{2}, \infty}}}
$$


where $\omega_{\mathrm{TO} 1, \mathrm{SiO} 2}$ is the low-frequency phonon mode of $\mathrm{SiO}_{2}, \varepsilon_{\mathrm{InSe}}$ is the dielectric constant of InSe. The potential amplitude of the remote phonon is written as

$$
\frac{1}{\hat{\varepsilon}_{R P}}=\frac{1}{\varepsilon_{\mathrm{InSe}}+\varepsilon_{\mathrm{SiO}_{2}, \infty}}-\frac{1}{\varepsilon_{\mathrm{InSe}}+\varepsilon_{\mathrm{SiO}_{2}, 0}}
$$

For the remote phonon induced by top gate dielectric, high- $\kappa$ dielectric covered with a metal gate is employed in the simulation. As shown in Table 1, the frequencies of two polar phonons in high- $\kappa$ dielectrics such $\mathrm{Al}_{2} \mathrm{O}_{3}$ and $\mathrm{HfO}_{2}$ show great discrepancy. Hence, for simplicity, only the low-frequency phonon mode in high- $\kappa$ gate dielectric is considered [19]. For high- $\kappa$ dielectric with a metal gate as shown in Figure 1b, the dispersion relationship is

$$
\omega_{R P}=\omega_{T O 1, H K} \frac{\left(\varepsilon_{\operatorname{InSe}}\left(\frac{1-e^{-2 q T}+e^{-2 q T}}{1+e^{-2 K}}\right)+\varepsilon_{H K, 0}\right)^{1 / 2}}{\left(\varepsilon_{\operatorname{InSe}}\left(\frac{1-e^{-2 q T} T_{K}}{1+e^{-2 q T} H K}\right)+\varepsilon_{H K, \text { int }}\right)^{1 / 2}}
$$

where $\omega_{T O 1, H K}$ is the low-frequency phonon mode of high- $\kappa$ dielectric, $\varepsilon_{H K, 0}$ and $\varepsilon_{H K, \text { int }}$ are the dielectric constant at the static and intermediate frequency, $T_{H K}$ is the thickness of top gate dielectric, and $q=\mid k$ $-k^{\prime} \mid$ is the remote phonon momentum. The effective dielectric constant depending on the frequency dependent dielectric constant of the high- $\kappa$ material can be written as

$$
\varepsilon_{e f f}=\varepsilon_{H K}(\omega) \frac{1+e^{-2 q T_{H K}}}{1-e^{-2 q T_{H K}}}+\varepsilon_{\text {InSe }}
$$

and then the corresponding potential amplitude is

$$
\frac{1}{\hat{\varepsilon}_{R P}}=\frac{1}{\varepsilon_{e f f}\left(\varepsilon_{H K, \text { int }}\right)}-\frac{1}{\varepsilon_{e f f}\left(\varepsilon_{H K, 0}\right)}
$$

For the high- $\kappa$ gate stack with a $\mathrm{SiO}_{2}$ interfacial layer, namely ITL/high- $\kappa /$ metal-gate stack as shown in Figure 1c, the dispersion relationship is [16]

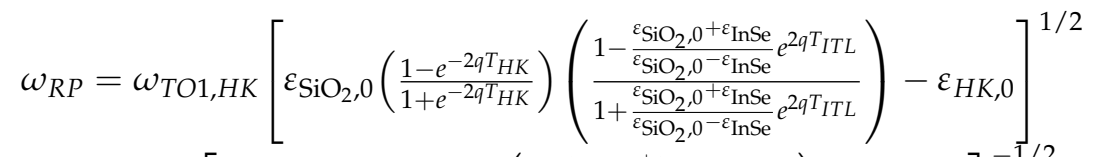

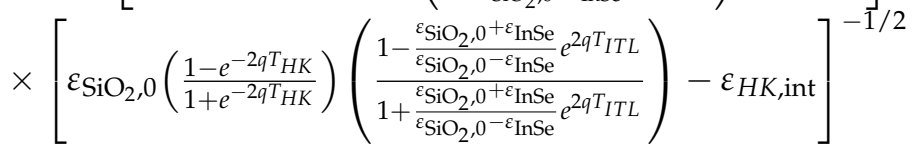

where $T_{I T L}$ is the thickness of interfacial layer. The effective dielectric constant is

$$
\begin{gathered}
\varepsilon_{e f f}(\omega)=\varepsilon_{H K}(\omega)\left[\left(\frac{\varepsilon_{\mathrm{SiO}_{2}, 0}-\varepsilon_{\mathrm{InSe}}}{2 \varepsilon_{\mathrm{SiO}_{2}, 0}}\right)^{2} e^{-2 q T_{I T L}}+\left(\frac{\varepsilon_{\mathrm{SiO}_{2}, 0}+\varepsilon_{\mathrm{InSe}}}{\varepsilon_{\mathrm{SiO}_{2}, 0}}\right)^{2} e^{2 q T_{I T L}}+2 \frac{\varepsilon_{\mathrm{SiO}_{2}, 0}^{2}-\varepsilon_{\mathrm{InSe}}^{2}}{\left(2 \varepsilon_{\mathrm{SiO}_{2}, 0}\right)^{2}}\right] \cdot \frac{1+e^{-2 q T} H K}{1-e^{-2 q T} T_{K}} \\
+\frac{\left(\varepsilon_{\mathrm{SiO}_{2}, 0}+\varepsilon_{\mathrm{InSe}_{\mathrm{IS}}}\right)^{2}}{4 \varepsilon_{\mathrm{SiO}_{2}, 0}} e^{2 q T_{I T L}}-\frac{\left(\varepsilon_{\mathrm{SiO}_{2}, 0}-\varepsilon_{\mathrm{InSe}}\right)^{2}}{4 \varepsilon_{\mathrm{Si}_{2}, 0}} e^{-2 q T_{I T L}}
\end{gathered}
$$

Then the potential amplitude for the ITL/high- $\kappa /$ metal-gate stack can be obtained through the Equations (5) and (7).

\section{Results and Discussion}

To begin with, we calibrate the physical models with the experimental measurement. Figure 2 shows the calculated and experimental temperature-dependent mobility at inversion density of $1.6 \times 10^{12} \mathrm{~cm}^{-2}$ and $7.9 \times 10^{12} \mathrm{~cm}^{-2}$ in six-layer InSe dual-gate FET. It should be noted that the experiment results are obtained from the dual-gate InSe FET with channel covered by hexagonal boron 
nitride (hBN) [9], which insulates InSe from the dielectric environment, leading to the absence of remote phonon scattering. From Figure $2 \mathrm{a}$, considering the intrinsic scatterings by AC, HO, and OP phonon and Fröhlich interaction, the temperature-dependent electron mobility curves measured by Hall effect are reproduced successfully for $\mathrm{T}>100 \mathrm{~K}$, where phonon scatterings dominate. The excellent agreement between the simulations and experiments validate our methods and models. It should be pointed out that when temperature is down to $100 \mathrm{~K}$, there is a significant discrepancy of mobility between simulations and experiments due to the fact that Coulomb scattering resulting from the channel impurities and interfacial charges is excluded, which is a dominant factor in determining the carrier mobility in the low-temperature regime.

On the other hand, if high- $\kappa$ dielectric of $\mathrm{HfO}_{2}$ is directly deposited on the InSe channel, the mobility is severely degraded from its intrinsic value, as shown in Figure 2a by solid lines. For example, at room temperature, mobility changes from 1808 to $1120 \mathrm{~cm}^{2} / \mathrm{Vs}$ (920 to $\left.464 \mathrm{~cm} / \mathrm{Vs}\right)$ at inversion density of $7.9 \times 10^{12} \mathrm{~cm}^{-2}\left(1.6 \times 10^{12} \mathrm{~cm}^{-2}\right)$ due to the remote phonon scattering. To understand the mobility behavior in depth, Figure $2 b, c$ plot the contributions of all the considered scattering mechanisms to the total mobility. Compared with AC, OP, and HO phonons, the remote phonon together with Fröhlich interaction plays a comparatively major role in determining the electron transport in InSe FET. This is the objective of this work to focus on the remote phonon scattering in InSe FET with high- $\kappa$ gate stack in the following.
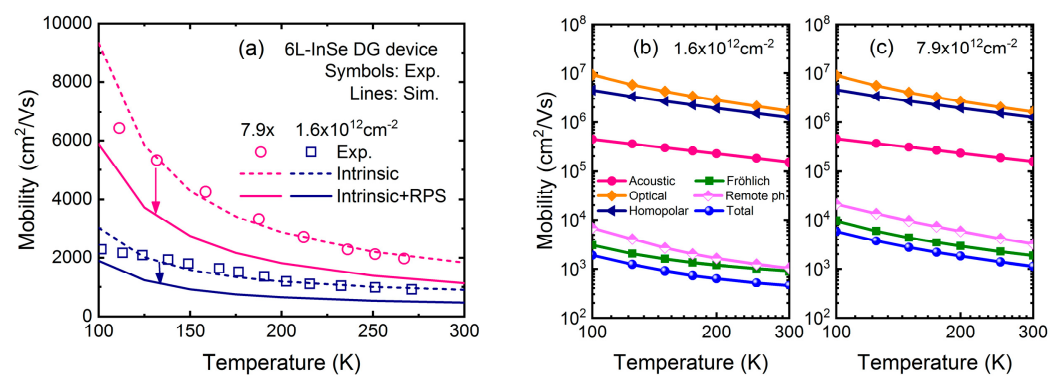

Figure 2. Temperature-dependent mobility in six-layer InSe FETs at inversion density of $1.6 \times 10^{12} \mathrm{~cm}^{-2}$ and $7.9 \times 10^{12} \mathrm{~cm}^{-2}$, respectively. (a) Comparison between the experimental mobility (symbols) and calculated intrinsic mobility without remote phonon scattering (RPS) (dashed lines), which shows an excellent agreement. In contrast, the mobility with RPS (solid lines) is significantly degraded. $(\mathbf{b}, \mathbf{c})$ Contributions of each scattering mechanisms to the total mobility for different inversion density respectively. Intrinsic scatterings include acoustic (AC), optical (OP), and homopolar (HO) phonon scatterings as well as Fröhlich interaction, while extrinsic scattering is remote phonon scattering arising from the $\mathrm{HfO}_{2}$ high- $\kappa$ dielectric.

The effect of remote phonon originating from the substrate and gate stack on the electron transport of few-layer InSe is shown in Figure 3. The intrinsic phonon-limited mobility in six-layer single-gate InSe FET is $\sim 843 \mathrm{~cm}^{2} / \mathrm{Vs}$ at low inversion density. With $\mathrm{SiO}_{2}$ substrate employed, the mobility is degraded to $\sim 735 \mathrm{~cm}^{2} / \mathrm{Vs}$ due to remote phonon. In the case of dual-gate structure, when $\mathrm{Al}_{2} \mathrm{O}_{3}$ and $\mathrm{HfO}_{2}$ are used as top-gate dielectric, the additional remote phonon further reduces the mobility to $\sim 634 \mathrm{~cm}^{2} / \mathrm{Vs}$ and $426 \mathrm{~cm}^{2} / \mathrm{Vs}$ respectively. It can be seen that the $\mathrm{HfO}_{2}$ dielectric has a much stronger influence of remote phonon than $\mathrm{Al}_{2} \mathrm{O}_{3}$ and $\mathrm{SiO}_{2}$ dielectric since it has higher dielectric constant and softer polar vibration mode [20], as listed in Table 1. Particularly, it is worth noting that the smaller permittivity of InSe results in a stronger remote phonon coupling with electrons compared with silicon even in the $\mathrm{SiO}_{2}$ case. Despite serious degradation due to remote phonons, the mobility of the six-layer InSe with high- $\kappa$ dielectric is higher than that of silicon on insulator (SOI) device with $\mathrm{SiO}_{2}$ dielectric at a comparative thickness [24], revealing its great potential in high-performance logic application. 


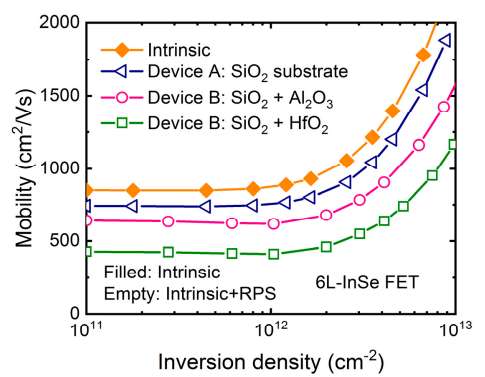

Figure 3. Calculated mobility as a function of inversion density in six-layer InSe FETs with different device structures as shown in Figure $1 \mathrm{a}$,b utilizing $\mathrm{Al}_{2} \mathrm{O}_{3}$ and $\mathrm{HfO}_{2}$ as top-gate dielectric and $\mathrm{SiO}_{2}$ substrate as back-gate dielectric respectively. Equivalent oxide thickness (EOT) $=1 \mathrm{~nm}$ of high- $\kappa$ dielectric is assumed. Filled symbols represent intrinsic phonon-limited mobility for benchmark, while empty symbols represent total mobility including the remote phonon scattering from substrate and high- $\kappa$ dielectric.

It is also observed that in six-layer InSe, mobility is increased significantly at higher density, which is against the common sense. To confirm this behavior, mobility in 2-, 6-, 16-, and 40-layer InSe FET is calculated in Figure $4 a$,b, where $\mathrm{HfO}_{2}$ and $\mathrm{Al}_{2} \mathrm{O}_{3}$ dielectric are used separately. At the same time, mobility in $\mathrm{MoS}_{2}$ FET using same device structure is also plotted in Figure 4c,d for comparison. In the $\mathrm{MoS}_{2}$ case, as inversion density increases, mobility monotonously decreases for thick devices as expected, and remains almost unchanged for thin devices due to strong quantum confinement. In the InSe case, at low density, mobility behavior is consistent with $\mathrm{MoS}_{2}$. However, when inversion density is larger than $\sim 2 \times 10^{12} \mathrm{~cm}^{-2}$, mobility quickly increases regardless of layer number or high- $\kappa$ dielectric. Actually, this is also demonstrated by experimental results in [9] as shown in Figure 2a, which cannot be totally contributed to impurity scattering because at large inversion density and room temperature the screening produced by the inversion layer drastically reduce the Coulomb scattering. Therefore, this exceptional enhancement seems intrinsic for 2D-layered InSe to a great extent.
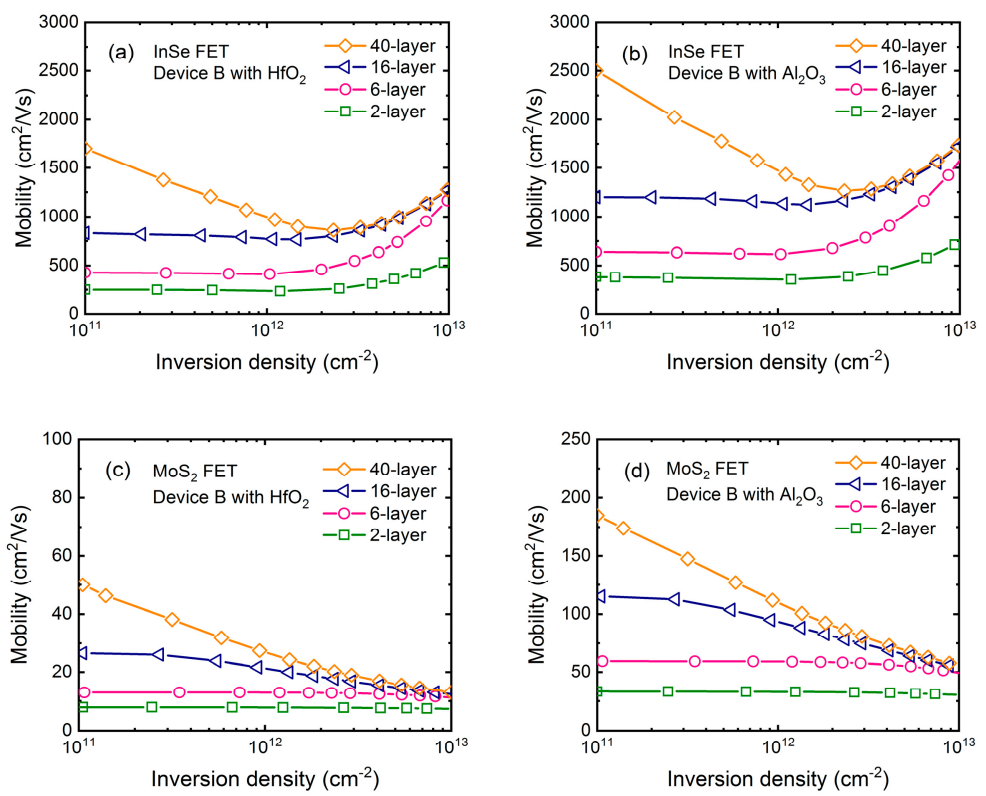

Figure 4. Mobility as a function of inversion density with layer number of 2,6, 16, and 40 employing the dual-gate structure as shown in Figure $1 \mathrm{~b}$ in InSe and $\mathrm{MoS}_{2}$ FET for comparison. EOT $=1 \mathrm{~nm}$ of high- $\kappa$ dielectric is assumed. (a) InSe FET with $\mathrm{HfO}_{2}$ dielectric. (b) InSe FET with $\mathrm{Al}_{2} \mathrm{O}_{3}$ dielectric. (c) $\mathrm{MoS}_{2}$ FET with $\mathrm{HfO}_{2}$ dielectric. (d) $\mathrm{MoS}_{2}$ FET with $\mathrm{Al}_{2} \mathrm{O}_{3}$. 
To get physical insight into this mobility behavior, Figure 5a-d shows the contributions of each scattering mechanism to the total mobility in both InSe and $\mathrm{MoS}_{2}$ FET with 2- and 40-layer thickness respectively. Consistent with above-mentioned results, mobility behavior is mainly governed by the remote phonon and Fröhlich interaction in all considered devices. We find that their scattering rates increase with inversion density increasing, which should reduce the mobility. In the $\mathrm{MoS}_{2}$ case, carriers are always non-degenerate following the Boltzmann distribution, where the mobility is essentially determined by the relaxation times or scattering rates. On the other hand, when inversion density is larger than $\sim 2 \times 10^{12} \mathrm{~cm}^{-2}$, carriers in InSe FET become degenerate, where subband minimum is lower than Fermi level $E_{F}$, and consequently the most influential relaxation times are those for energies close to $E_{F}$. Due to their anisotropic property, scattering rates of remote phonon and Fröhlich interaction are much smaller near $E_{F}$ than those of the subband minimum, giving rise to an enhancement of mobility. This discrepancy between InSe and $\mathrm{MoS}_{2}$ FET can be well understood by their effective masses. Firstly, in-plane effective mass of $0.14 m_{0}$ in InSe is much smaller than $0.62 m_{0}$ in $\mathrm{MoS}_{2}$ [25] leading to much smaller density-of-states (DOS). In order to obtain the same density, the conduction band minimum is lower than Fermi level over a few $k_{\mathrm{B}} T$, where $k_{\mathrm{B}}$ is the Boltzmann constant. Secondly, quantization effective mass of $0.08 m_{0}$ in InSe is also much smaller than $0.49 m_{0}$ in $\mathrm{MoS}_{2}$ [26]. This is the reason that quantum confinement takes effect in 16-layer InSe, but not until the layer number is reduced to 6 layers in $\mathrm{MoS}_{2}$. Actually, less subbands contributing to the carrier transport in InSe need Fermi level being higher to change the density, which makes carriers more degenerate together with the effect of small DOS.
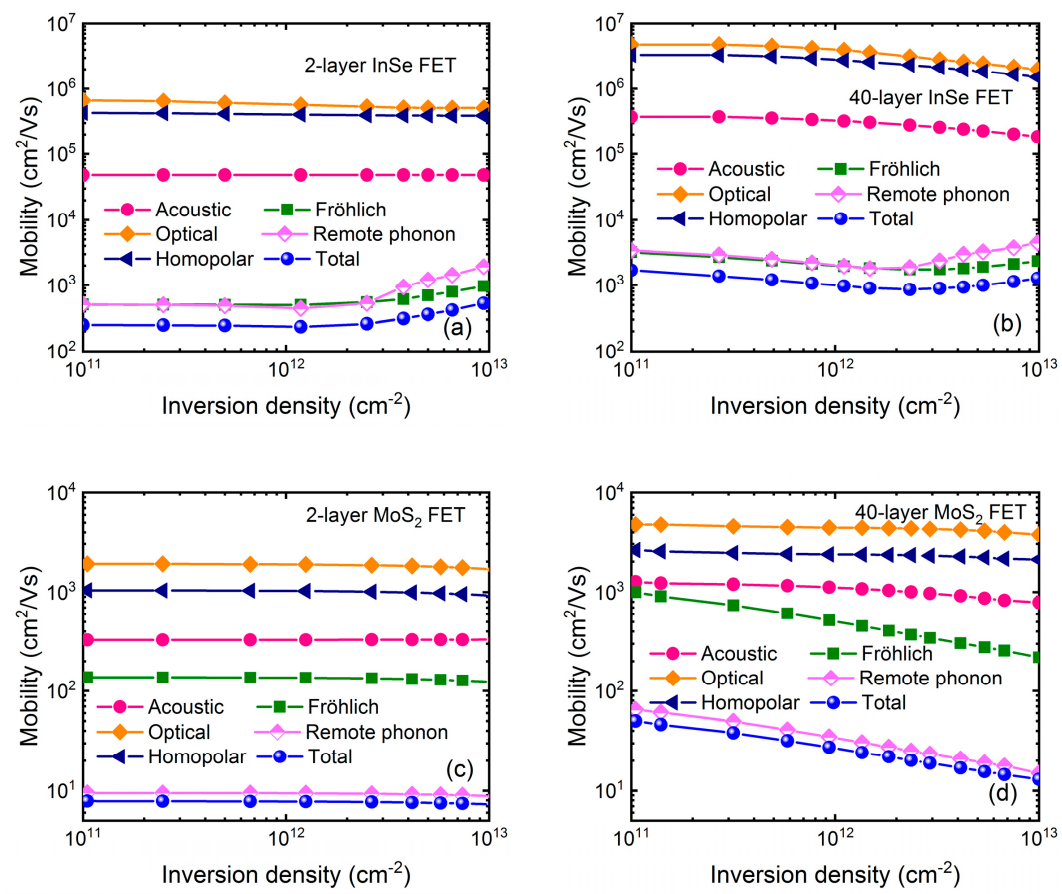

Figure 5. Contributions of each scattering process to the total mobility corresponding to Figure $4 \mathrm{a}, \mathrm{c}$ for InSe and $\mathrm{MoS}_{2}$ FET with $\mathrm{HfO}_{2}$ dielectric respectively. (a) 2-layer InSe FET. (b) 40-layer InSe FET. (c) 2-layer $\mathrm{MoS}_{2}$ FET. (d) 40-layer $\mathrm{MoS}_{2}$ FET.

The dependence of mobility on number of layer (NL) in InSe FET is shown in Figure 6a, where inversion densities of $5 \times 10^{11}, 2 \times 10^{12}$, and $8 \times 10^{12} \mathrm{~cm}^{-2}$ are considered respectively. In the case of the relatively medium and high density, mobility is almost independent of channel thickness until NL $\sim 15$, and then drops rapidly as NL is further reduced. In the low-density case, mobility degradation occurs earlier when NL $<40$. It is noted that for thicker devices, mobility at $5 \times 10^{11} \mathrm{~cm}^{-2}$ gradually surpasses the value at $2 \times 10^{12} \mathrm{~cm}^{-2}$ and then reaches up to the value at $8 \times 10^{12} \mathrm{~cm}^{-2}$. This awkward 
behavior can be explained by Figure $4 a, b$, where mobility initially decrease and then the trend is opposite at higher density, as inversion density decreases in the 40-layer InSe FET. To get physical insight into the mobility degradation, contributions of each scattering mechanism at $5 \times 10^{11} \mathrm{~cm}^{-2}$ is shown in Figure 6b. AC-, LO-, and HO-limited mobility is severely reduced when NL is less than $\sim 15$, while the degradation of remote-phonon as well as Fröhlich-limited mobility begins to decrease about NL $\sim 40$, further indicating their major role in determining the electron transport.
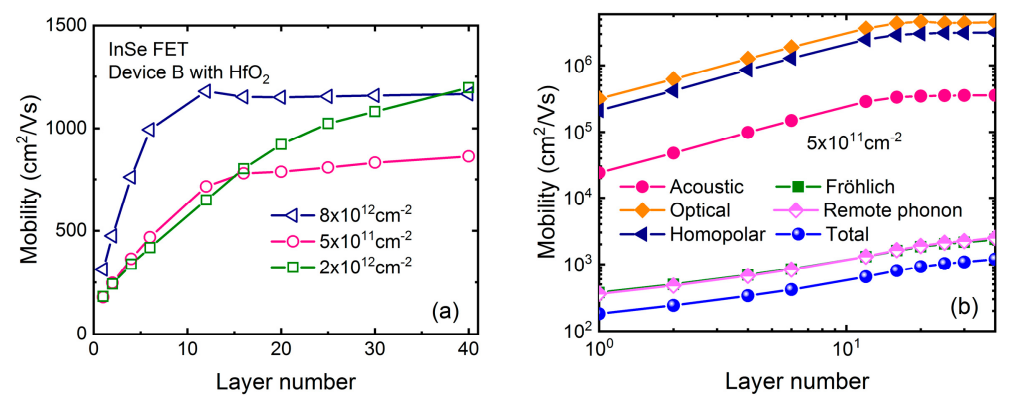

Figure 6. (a) Mobility as a function of number of layers ranging from 1 to 40 with different inversion density in InSe FET using $\mathrm{HfO}_{2}$ as gate dielectric and $\mathrm{SiO}_{2}$ as substrate, as shown in Figure $1 \mathrm{~b}$.

(b) Contributions of each scattering mechanism to the total mobility at low inversion density.

Inspired by the similar situation for silicon $[27,28]$, it is suggested that an interfacial layer can be introduced between InSe channel and high- $\kappa$ dielectric. To explore the effect of the interfacial layer, Figure 7 shows the calculated mobility in InSe FET with device structure of Figure 1c capped with different high- $\kappa$ dielectric. From Figure $7 a$, it can be seen that the interfacial layer effectively insulates the channel away from the high- $\kappa$ dielectric, resulting in significant mobility enhancement in the whole range of inversion density due to weaker remote phonon coupling. As interfacial layer thickness is increased, more mobility enhancement is achieved until $\mathrm{T}_{\mathrm{ITL}}$ approaches $\sim 2 \mathrm{~nm}$, when remote phonon from high- $\kappa$ dielectric is totally separated, as shown in Figure $7 \mathrm{~b}$. Besides, a thin interfacial layer is more effective in $\mathrm{HfO}_{2}$ dielectric compared with $\mathrm{Al}_{2} \mathrm{O}_{3}$ dielectric. Figure 8 shows the corresponding remote coulomb scattering (RCS)-limited mobility as a function of $\mathrm{SiO}_{2}$ interfacial layer thickness, showing an exponential dependence on $\mathrm{T}_{\mathrm{ITL}}$ as $\mu_{\mathrm{RPS}} \propto \exp \left(2 k_{\mathrm{F}} \mathrm{T}_{\mathrm{ITL}}\right)$, whatever the inversion density or high- $\kappa$ dielectric is, with Fermi wavelength $2 k_{\mathrm{F}}=1.1 \mathrm{~nm}^{-1}$. This is in agreement with theoretical predictions for a remote scattering mechanism $[20,28]$.
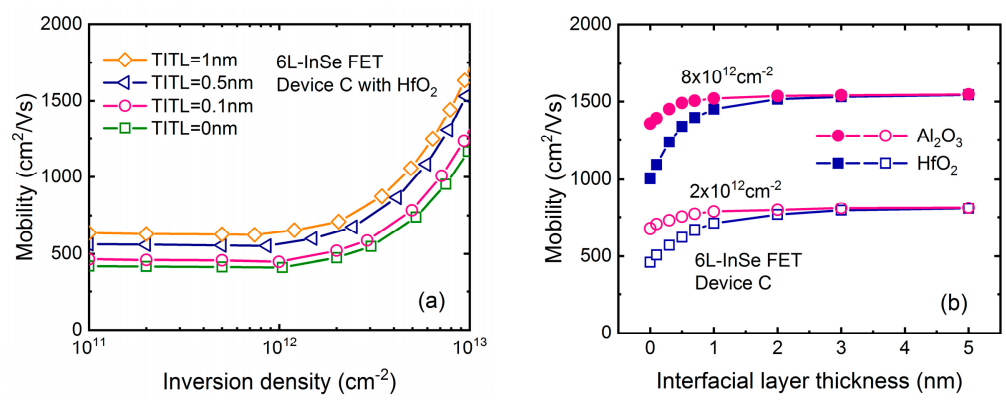

Figure 7. (a) Mobility as a function of inversion density featuring different $\mathrm{SiO}_{2}$ interfacial layer thickness in InSe FET with dual-gate structure of Figure 1c. (b) Mobility as a function of $\mathrm{SiO}_{2}$ interfacial layer thickness at different inversion density in both $\mathrm{Al}_{2} \mathrm{O}_{3}$ - and $\mathrm{HfO}_{2}$-gated InSe FET. EOT = $1 \mathrm{~nm}$ of high- $\kappa$ dielectric is used in the simulation. 


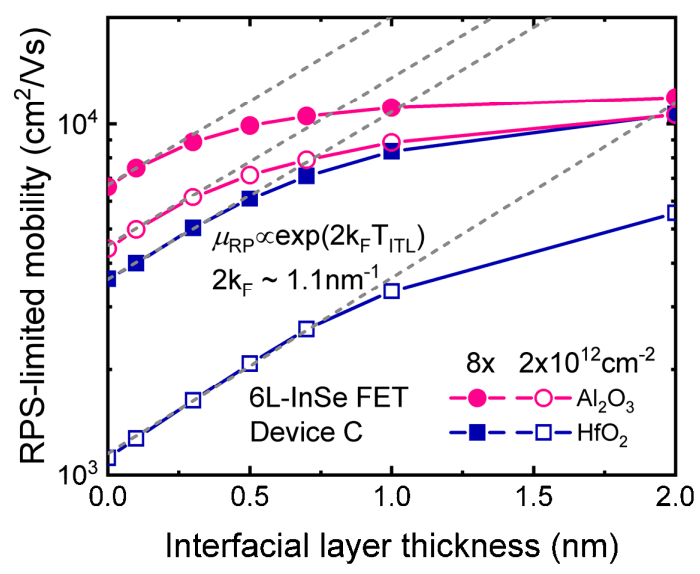

Figure 8. Remote coulomb scattering (RCS)-limited mobility as a function of $\mathrm{SiO}_{2}$ interfacial layer thickness corresponding to total mobility in Figure $7 \mathrm{~b}$, showing an exponential dependence on $\mathrm{T}_{\text {ITL }}$ as $\exp \left(2 k_{\mathrm{F}} \mathrm{T}_{\mathrm{ITL}}\right)$ with $2 k_{\mathrm{F}}=1.1 \mathrm{~nm}^{-1}$, represented by the dashed lines.

\section{Conclusions}

Based on the self-consistent Poisson and Schrödinger equations and the Kubo-Greenwood formula, remote phonons arising from both the $\mathrm{SiO}_{2}$ substrate and high- $\kappa$ dielectrics in InSe FETs are comprehensively studied, together with the intrinsic scatterings by AC phonons, OP phonons, HO phonons, and Fröhlich interaction. It is observed that remote phonons and Fröhlich interaction plays a comparatively major role in determining the electron transport in InSe. Mobility is more severely degraded by remote phonon of $\mathrm{HfO}_{2}$ dielectric than $\mathrm{Al}_{2} \mathrm{O}_{3}$ and $\mathrm{SiO}_{2}$ dielectric, which can be effectively insulated by introducing a $\mathrm{SiO}_{2}$ interfacial layer between the high- $\kappa$ dielectric and InSe. Due to its smaller in-plane and quantization effective masses, mobility begins to increase at higher densities as carriers degenerate, and mobility degradation with reduced layer number is much stronger in InSe compared with $\mathrm{MoS}_{2}$.

Author Contributions: Conceptualization, P.C.; Formal analysis, P.C.; Funding acquisition, P.C.; Investigation, P.C.; Methodology, P.C. and F.L.; Resources, X.L.; Software, P.C.; Supervision, X.L. and G.D.; Validation, P.C.; Writing-Original Draft, P.C.

Funding: This research was funded by China Postdoctoral Science Foundation (grant number 2018M630034) and National Natural Science Foundation of China (grant numbers 61804003, 61674008, 61421005).

Conflicts of Interest: The authors declare no conflict of interest.

\section{References}

1. Bernstein, K.; Cavin, R.K.; Porod, W.; Seabaugh, A.; Welser, J. Device and Architecture Outlook for Beyond CMOS Switches. Proc. IEEE 2010, 98, 2169-2184. [CrossRef]

2. Mistry, K.; Allen, C.; Auth, C.; Beattie, B.; Bergstrom, D.; Bost, M.; Brazier, M.; Buehler, M.; Cappellani, A.; Chau, R.; et al. A 45nm Logic Technology with High- $\kappa+$ Metal Gate Transistors, Strained Silicon, $9 \mathrm{Cu}$ Interconnect Layers, 193nm Dry Patterning, and 100\% Pb-free Packaging. In Proceedings of the IEEE International Electron Devices Meeting, Washington, DC, USA, 10-12 December 2007. [CrossRef]

3. Natarajan, S.; Agostinelli, M.; Akbar, S.; Bost, M.; Bowonder, A.; Chikarmane, V.; Chouksey, S.; Dasgupta, A.; Fischer, K.; Fu, Q.; et al. A 14nm Logic Technology Featuring 2nd-Generation FinFET Transistors, Air-Gapped Interconnects, Self-Aligned Double Patterning and a $0.0588 \mu \mathrm{m}^{2}$ SRAM cell size. In Proceedings of the IEEE International Electron Devices Meeting, San Francisco, CA, USA, 15-17 December 2014. [CrossRef]

4. Fiori, G.; Bonaccorso, F.; Iannaccone, G.; Palacios, T.; Neumaier, D.; Seabaugh, A.; Banerjee, S.K.; Colombo, L. Electronics based on two-dimensional materials. Nat. Nanotechnol. 2014, 9, 768-779. [CrossRef] [PubMed] 
5. Novoselov, K.S.; Mishchenko, A.; Carvalho, A.; Castro Neto, A.H. 2D materials and van der Waals heterostructures. Science 2016, 353, 439. [CrossRef] [PubMed]

6. Novoselov, K.S.; Geim, A.K.; Morozov, S.V.; Jiang, D.; Zhang, Y.; Dubonos, S.V.; Grigorieva, I.V.; Firsov, A.A. Electric Field Effect in Atomically Thin Carbon Films. Science 2004, 306, 666-669. [CrossRef]

7. Schmidt, H.; Wang, S.; Chu, L.; Toh, M.; Kumar, R.; Zhao, W.; Castro Neto, A.H.; Martin, J.; Adam, S.; Ozylimaz, B.; et al. Transport Properties of Monolayer $\mathrm{MoS}_{2}$ Grown by Chemical Vapor Deposition. Nano Lett. 2014, 14, 1909-1913. [CrossRef] [PubMed]

8. Li, L.; Yu, Y.; Ye, G.J.; Ge, Q.; Ou, X.; Wu, H.; Feng, D.; Cheng, X.H.; Zhang, Y. Black phosphorus field-effect transistors. Nat. Nanotechnol. 2014, 9, 372-377. [CrossRef] [PubMed]

9. Bandurin, D.A.; Tyurnina, A.V.; Yu, G.L.; Mishchenko, A.; Zólyomi, V.; Morozov, S.V.; Kumar, R.K.; Gorbachev, R.V.; Kudrynskyi, Z.R.; Pezzini, S.; et al. High Electron Mobility, Quantum Hall Effect and Anomalous Optical Response in Atomically Thin InSe. Nat. Nanotechnol. 2017, 9, 223-227. [CrossRef] [PubMed]

10. Feng, W.; Zheng, W.; Cao, W.; Hu, P. Back Gated Multilayer InSe Transistors with Enhanced Carrier Mobilities via the Suppression of Carrier Scattering from a Dielectric Interface. Adv. Mater. 2014, 26, 6587-6593. [CrossRef] [PubMed]

11. Sucharitakul, S.; Goble, N.J.; Kumar, U.R.; Sankar, R.; Bogorad, Z.A.; Chou, F.-C.; Chen, Y.-T.; Gao, X.P.A. Intrinsic Electron Mobility Exceeding $10^{3} \mathrm{~cm}^{2} /(\mathrm{Vs})$ in Multilayer InSe FETs. Nano Lett. 2015, 15, 3815-3819. [CrossRef]

12. Premasiri, K.; Radha, S.K.; Sucharitakul, S.; Kumar, U.R.; Sankar, R.; Chou, F.-C.; Chen, Y.-T.; Gao, X.P.A. Tuning Rashba Spin-Orbit Coupling in Gated Multilayer InSe. Nano Lett. 2018, 18, 4403-4408. [CrossRef]

13. Zeng, J.; Liang, S.-J.; Gao, A.; Wang, Y.; Pan, C.; Wu, C.; Liu, E.; Zhang, L.; Cao, T.; Liu, X.; et al. Gate-tunnable weak antilocalization in a few-layer InSe. Phys. Rev. B 2018, 98, 125414. [CrossRef]

14. Chang, P.; Liu, X.; Liu, F.; Du, G. First-principles based ballistic transport simulation of monolayer and few-layer InSe FET. Jpn. J. Appl. Phys. 2018. under review.

15. Chang, P.; Liu, X.; Liu, F.; Du, G. Phonon-Limited Mobility in n-Type Few-Layer InSe Devices from First Principles. IEEE Electron Devices Lett. 2018. [CrossRef]

16. Li, S.-L.; Tsukagoshi, K.; Orgiu, E.; Samori, P. Charge transport and mobility engineering in two-dimensional transition metal chalcogenide semiconductors. Chem. Soc. Rev. 2016, 45, 118-151. [CrossRef] [PubMed]

17. Li, S.-L.; Wakabayashi, K.; Xu, Y.; Nakaharai, S.; Komatsu, K.; Li, W.-W.; Lin, Y.-F.; Ferreira, A.A.; Tsukagoshi, K. Thickness-Dependent Interfacial Coulomb Scattering in Atomically Thin Field-Effect Transistors. Nano Lett. 2013, 13, 3546-3552. [CrossRef]

18. Zeng, L.; Xin, Z.; Chen, S.; Du, G.; Kang, J.; Liu, X. Remote phonon and impurity screening effect of substrate and gate dielectric on electron dynamics in single layer $\mathrm{MoS}_{2}$. Appl. Phys. Lett. 2013, 103, 113505. [CrossRef]

19. Esseni, D.; Palestri, P.; Selmi, L. Nanoscale MOS Transistors: Semi-Classical Transport and Applications; Cambridge University Press: New York, NY, USA, 2011; pp. 98-103.

20. Fischetti, M.V.; Neumayer, D.A.; Cartier, E.A. Effective electron mobility in Si inversion layers in metal-oxide-semiconductor systems with a high-k insulator: The role of remote phonon scattering. J. Appl. Phys. 2001, 90, 4587-4606. [CrossRef]

21. Poljak, M.; Jovanovié, V.; Grgec, D.; Suligoj, T. Assessment of Electron Mobility in Ultrathin-Body InGaAs-on-Insulator MOSFETs Using Physics-Based Modeling. IEEE Trans. Electron Devices 2012, 59, 1636-1643. [CrossRef]

22. Chang, P.; Liu, X.; Zeng, L.; Wei, K.; Du, G. Investigation of Hole Mobility in Strained InSb Ultrathin Body pMOSFETs. IEEE Trans. Electron Devices 2015, 62, 947-954. [CrossRef]

23. Chang, P.; Liu, X.; Du, G.; Zhang, X. Assessment of Hole Mobility in Strained InSb, GaSb and InGaSb Based Ultra-Thin Body pMOSFETs with Different Surface Orientations. In Proceedings of the IEEE International Electron Devices Meeting, San Francisco, CA, USA, 15-17 December 2014. [CrossRef]

24. Tsutsui, G.; Hiramoto, T. Mobility and Threshold-Voltage Comparison Between (110)- and (100)-Oriented Ultrathin-Body Silicon MOSFETs. IEEE Trans. Electron Devices 2006, 53, 2582-2588. [CrossRef] 
25. Gonzalez-Medina, J.M.; Ruiz, F.G.; Martin, E.G.; Godoy, A.; Gámiz, F. Simulation study of the electron mobility in few-layer $\mathrm{MoS}_{2}$ metal-insulator-semiconductor field-effect transistors. Solid-State Electron. 2015, 114, 30-34. [CrossRef]

26. Segura, A.; Marí, B.; Martinez-Pastor, J. Three-dimensional electrons and two-dimensional electric subbands in the transport properties of tin-doped n-type indium selenide: Polar and homopolar phonon scattering. Phys. Rev. B 1991, 43, 4953-4965. [CrossRef]

27. Chau, R.; Datta, S.; Doczy, M.; Doyle, B.; Kavalieros, J.; Metz, M. High- $\kappa /$ Metal-Gate Stack and Its MOSFET Characteristics. IEEE Electron Device Lett. 2004, 6, 408-410. [CrossRef]

28. Cassé, M.; Thevenod, L.; Guillaumot, B.; Tosti, L.; Martin, F.; Mitard, J.; Weber, O.; Andrieu, F.; Ernst, T.; Reimbold, G.; et al. Carrier Transport in $\mathrm{HfO}_{2} /$ Metal Gate MOSFETs: Physical Insight into Critical Parameters. IEEE Trans. Electron Devices 2006, 53, 759-768. [CrossRef]

(C) 2018 by the authors. Licensee MDPI, Basel, Switzerland. This article is an open access article distributed under the terms and conditions of the Creative Commons Attribution (CC BY) license (http://creativecommons.org/licenses/by/4.0/). 